Pacific

Journal of

Mathematics

HOMOTOPICALLY PERIODIC MAPS OF MODEL ASPHERICAL MANIFOLDS

Wim Malfait

Volume 199 No. 1

May 2001 


\title{
HOMOTOPICALLY PERIODIC MAPS OF MODEL ASPHERICAL MANIFOLDS
}

\author{
Wim MALFAit
}

\begin{abstract}
For a closed orientable surface $S$, any map $f: S \rightarrow S$ whose $n$-th power is homotopic to the identity, is homotopic to a homeomorphism $g$ of $S$ of order $n$. This famous theorem of Nielsen is known to fail in general for aspherical manifolds. In this paper, for model aspherical manifolds $M$ associated to a finitely extendable set of data, we, however, present a weaker version of Nielsen's result. We show that any homotopically periodic self-map $f$ of $M$ is homotopic to a fiber preserving homeomorphism $g$ of $M$ of finite order (but the order of $g$ is not necessarily equal to the homotopy period of $f$ ).
\end{abstract}

\section{Introduction.}

In this paper, we extend and complement the results of [9] on the validity of Nielsen's theorem for manifolds $M$ which arise from a (generalized) Seifert fiber space construction (the so-called model aspherical manifolds). The study of these manifolds is of major importance since, at this moment, there are no aspherical manifolds known which can not be constructed in this way.

Let us recall the main theorem of [9]: Under some additional conditions on the model aspherical manifold $M$, a finite abstract kernel $G \rightarrow \operatorname{Out}\left(\pi_{1}(M)\right)$ can be realized as an (effective) action of $G$ on $M$ via fiber preserving homeomorphisms if and only if there is an (admissible) group extension compatible with $\psi$. We reformulate this result here (Theorem 3.5) in a more comprehensible way.

In other words, the obstruction for finite abstract kernels $\psi: G \rightarrow$ $\operatorname{Out}\left(\pi_{1}(M)\right)$ to admit a geometric realization by a group of fiber preserving homeomorphisms of $M$, is the failure of the existence of an extension realizing $\psi$. This can indeed happen when the center of $\pi_{1}(M)$ is not trivial (explicit examples are given in [2], [7], [10], [11]).

In the sequel, we show, for an interesting subclass of model aspherical manifolds $M$, that for any (faithful) finite abstract kernel $\psi: G \rightarrow$ $\operatorname{Out}\left(\pi_{1}(M)\right)$, there is an epimorphism $H \rightarrow G$ of a finite group $H$ such that $H \rightarrow G \stackrel{\psi}{\rightarrow} \operatorname{Out}\left(\pi_{1}(M)\right)$ admits an (effective) geometric realization by a group of fiber preserving homeomorphisms of $M$ (Theorem 3.6). 
Although this observation is already interesting in itself, it is the key result which allows to prove that each homotopically periodic self-map $f$ of $M$ is homotopic to a fiber preserving homeomorphism $g$ of $M$ of finite order (Theorem 3.7). As we will show and illustrate, the order of $g$, say $l$, can be strictly greater than the homotopy period $k$ of $f$ but, if $n$ denotes the rank of the center of $\pi_{1}(M)$, then $l$ always divides $k^{n+1}$. This seems to be the best possible way to generalize Nielsen's theorem towards (model) aspherical manifolds.

It is important to note that the results presented here hold for all model aspherical manifolds already described in the literature (flat Riemannian manifolds, infra-nilmanifolds, infra-solvmanifolds of type $(\mathrm{R})$ ).

\section{Model aspherical manifolds.}

An aspherical manifold $M$ is a closed connected manifold whose universal covering is contractible. An aspherical manifold is a $K(E, 1)$-space: All higher homotopy groups are trivial but the fundamental group $\pi_{1}(M)$ is isomorphic to $E$. An interesting class of aspherical manifolds are those arising from a Seifert fiber space construction and are referred to as model aspherical manifolds. Let us briefly review the main ideas of this set-up. For more details and background, we refer to [9] and the references there.

Let $L$ be a connected, simply connected Lie group, $N$ is a lattice of $L$ (i.e., a discrete subgroup of $L$ such that $L / N$ is compact) and each automorphism of $N$ extends uniquely to an automorphism of $L$ (we say $(N, L)$ has UAEP). Assume $W$ is a connected, simply connected topological space such that $L \times W$ is a manifold. Write $\mathcal{H}(W)$ for the group of homeomorphisms of $W$ and $\mathcal{M}\left(W, L^{*}\right)$ for the group of continuous mappings $W \rightarrow L$ with multiplication: $\left(\lambda_{1} * \lambda_{2}\right)(w)=\lambda_{2}(w) \cdot \lambda_{1}(w)$. Then $\operatorname{Aut}(L) \times \mathcal{H}(W)$ acts on $\mathcal{M}\left(W, L^{*}\right)$ and defines a semi-direct product $\mathcal{H}^{f}(L \times W)=\mathcal{M}\left(W, L^{*}\right) \rtimes$ (Aut $(L) \times \mathcal{H}(W))$. This is a transformation group of $L \times W$ and each element of $\mathcal{H}^{f}(L \times W)$ preserves the fibers of $L \rightarrow L \times W \rightarrow W$. The Lie group $L$ embeds into $\mathcal{M}\left(W, L^{*}\right) \rtimes \operatorname{Inn}(L)$ via $l: L \rightarrow \mathcal{H}^{f}(L \times W): x \mapsto(x, \mu(x), 1)$ and acts on $L \times W$ as (left) translations in the $L$-factor. One verifies that $\mathcal{H}^{f}(L \times W)$ normalizes $l(L)$ and therefore $\mathcal{H}^{f}(L \times W)$ is referred to as the group of fiber preserving homeomorphisms of $L \times W$ compatible with left translations.

We say that a properly discontinuous action $\rho: F \rightarrow \mathcal{H}(W)$ of a group $F$ on $W$ has the $L$-Seifert property if and only if for every extension $1 \rightarrow L \rightarrow$ $\mathcal{E} \rightarrow F \rightarrow 1$ (inducing an abstract kernel $\psi: F \rightarrow \operatorname{Out}(L)$ ), there exists a homomorphism $\hat{\Psi}$ making the following diagram commutative

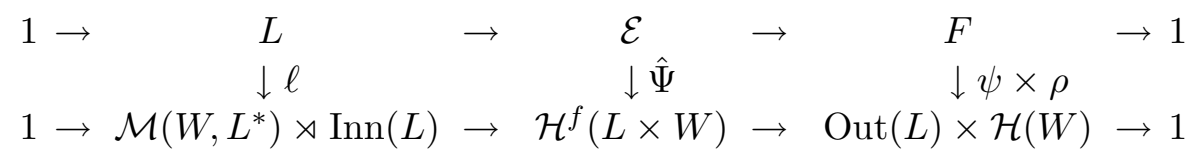


A quadruple $(N, E, \rho, W)$ is an $L$-set of data if and only if $N$ is a lattice in $L$ such that $(N, L)$ has UAEP and $N$ is a normal subgroup of $E$ such that $\rho: F=E / N \rightarrow \mathcal{H}(W)$ is a properly discontinuous action of $F$ on $W$ having the $L$-Seifert property. Well-known examples are:

1) If $L$ is nilpotent and $N$ is a lattice of $L$, then for each extension $1 \rightarrow$ $N \rightarrow E \rightarrow F \rightarrow 1$ and properly discontinuous action $\rho$ of $F$ on $W$, $(N, E, \rho, W)$ is an $L$-set of data $([3])$.

2) If $L$ is solvable with connected center and $N$ is a lattice in $L$ such that $(N, L)$ has UAEP, then for every extension $1 \rightarrow N \rightarrow E \rightarrow F \rightarrow 1$ and properly discontinuous action $\rho$ of $F$ on $W,(N, E, \rho, W)$ is an $L$-set of data $([8])$.

Given an $L$-set of data $(N, E, \rho, W)$, there always exists a homomorphism $\Psi: E \rightarrow \mathcal{H}^{f}(L \times W)$ making the following diagram commutative:

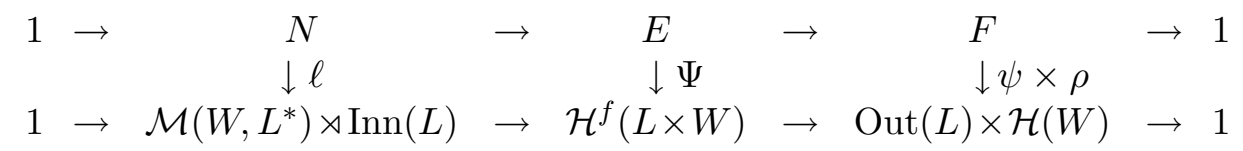

$(\psi: F \rightarrow \operatorname{Out}(L)$ is induced by $1 \rightarrow N \rightarrow E \rightarrow F \rightarrow 1$ and the UAEP of $(N, L))$. We refer to $\Psi: E \rightarrow \mathcal{H}^{f}(L \times W)$ as a Seifert construction for the set of data $(N, E, \rho, W)$. This concept has, besides existence, also uniqueness and rigidity as important properties but, as those are not of primary importance here, we refer the reader to $[\mathbf{9}]$.

The action of $E$ on $L \times W$, via this $\Psi: E \rightarrow \mathcal{H}^{f}(L \times W)$ is properly discontinuous. Moreover, if we suppose that $E$ is torsion-free, then $E$ acts freely and effectively on $L \times W$ (via $\Psi$ ). Additionally, if we assume $W, L$ contractible and $F \backslash W$ compact, then such a set of data defines a $K(E, 1)$ manifold $M=E \backslash(L \times W)$. We refer to $M$ as the model aspherical manifold associated to (or modelled on) the $L$-set of data $(N, E, \rho, W)$.

Many families of aspherical manifolds studied in literature before, arise in this way. We refer to:

1) [6] for flat Riemannian manifolds: $L=\mathbb{R}^{k}\left(k \in \mathbb{N}_{0}\right), N=\mathbb{Z}^{k}$ is of finite index in $E$ ( $E$ is a Bieberbach group), $W$ is a point and $\rho$ is the trivial action;

2) [3] for infra-nilmanifolds: $L$ is nilpotent, $N$ is of finite index in $E$ ( $E$ is an almost-Bieberbach group), $W$ is a point and $\rho$ is the trivial action;

3) [5] for infra-solvmanifolds of type (R): $L$ is solvable of type (R), $N$ is of finite index in $E, W$ is a point and $\rho$ is the trivial action.

\section{Realizing a finite abstract kernel.}

For an aspherical manifold $M$, there are two possible approaches to the realization-problem of a finite abstract kernel $\psi: G \rightarrow \operatorname{Out}\left(\pi_{1}(M)\right)$. Firstly, 
there is the (well known) algebraic concept of realizing $\psi$ by a group extension and secondly, there is the geometric question if $\psi$ admits a realization by a group of homeomorphisms of $M$. We discuss both concepts and study how they are related to each other.

3.1. Algebraic approach. A group extension $1 \rightarrow E \rightarrow E^{\prime} \rightarrow G \rightarrow 1$ induces, by choosing a normalized section and via conjugation in $E^{\prime}$, a homomorphism $\psi: G \rightarrow \operatorname{Out}(E)=\operatorname{Aut}(E) / \operatorname{Inn}(E)$ which is called an abstract kernel. We say that $1 \rightarrow E \rightarrow E^{\prime} \rightarrow G \rightarrow 1$ realizes, or is compatible with, $\psi$. Obviously, the kernel of $\psi$ is exactly $C_{E^{\prime}}(E) / Z(E)$. An extension $1 \rightarrow E \rightarrow E^{\prime} \rightarrow G \rightarrow 1$ is admissible if and only if the centralizer $C_{E^{\prime}} E$ of $E$ in $E^{\prime}$ is torsion-free. So, an abstract kernel $\psi: G \rightarrow \operatorname{Out}(E)$ can be (admissibly) algebraically realized if and only if there exists an (admissible) extension $1 \rightarrow E \rightarrow E^{\prime} \rightarrow G \rightarrow 1$ compatible with $\psi$.

This realization-problem is well-known in the literature and is conceptually well-understood: There exists a group extension compatible with $\psi: G \rightarrow \operatorname{Out}(E)$ if and only if a well-determined cohomology class in $H^{3}(G, Z(E))$ vanishes. In general, this so-called obstruction, measuring the failure to realize $\psi$, is unfortunately extremely hard to compute. For finite cyclic groups however, there is the following nice criterion (which will be referred to in the sequel):

Lemma 3.1. Let $E$ be a group. Assume $\sigma \in \operatorname{Aut}(E)$ and, for some $k \in \mathbb{N}_{0}$, $\sigma^{k} \in \operatorname{Inn}(E)$. Then the induced abstract kernel $\psi: \mathbb{Z}_{k} \rightarrow \operatorname{Out}(E)$ can be realized by a group extension if and only if there exists an element $x \in E$ such that $\sigma^{k}=\mu(x)$ and $\sigma(x)=x$.

Inspired by this result (due to B. Zimmermann), in [10] we presented similar, also pure algebraic, criteria to decide if a given finite abstract kernel $\psi$ admits an extension compatible with $\psi$.

A way to overcome possible lack of existence of an algebraic realization is given by another result of Zimmermann:

Proposition $3.2([\mathbf{1 2}])$. Let $\psi: G \rightarrow \operatorname{Out}(E)$ be a finite abstract kernel and suppose $Z(E) \cong \mathbb{Z}^{n}$, for some $n \in \mathbb{N}$. Assume that the obstruction corresponding to $\psi$ is of order $k$ in $H^{3}(G, Z(E))$. Then there is a finite group $H$, fitting into an extension $1 \rightarrow\left(\mathbb{Z}_{k}\right)^{n} \rightarrow H \stackrel{j}{\rightarrow} G \rightarrow 1$, such that the abstract kernel $\psi \circ j: H \rightarrow \operatorname{Out}(E)$ can be algebraically realized.

Since $H$ is finite, it is always possible to reduce to a minimal inflation $j$ : $H \rightarrow G$ (terminology also due to Zimmermann). This means that $\psi \circ j: H \rightarrow$ $\operatorname{Out}(E)$ can be algebraically realized and there is no non-trivial subgroup $T$ of $H$ with $j(T)=\{1\}$ such that $\psi \circ j: H / T \rightarrow \operatorname{Out}(E)$ can be realized by an extension. 
Obviously, in case $\psi: G \rightarrow \operatorname{Out}(E)$ is a faithful, finite abstract kernel and if the center $Z(E)$ of $E$ is torsion-free, then each extension compatible with $\psi$ is admissible. We generalize this natural observation as follows:

Lemma 3.3. Let $\psi: G \rightarrow \operatorname{Out}(E)$ be a faithful, finite abstract kernel and suppose $Z(E) \cong \mathbb{Z}^{n}$, for some $n \in \mathbb{N}$. If $j: H \rightarrow G$ is a minimal inflation, then each extension realizing $\psi \circ j: H \rightarrow \operatorname{Out}(E)$ is admissible.

Proof. Assume $1 \rightarrow E \rightarrow E^{\prime} \rightarrow H \rightarrow 1$ realizes $\psi \circ j: H \rightarrow \operatorname{Out}(E)$. Since $E$ is a normal subgroup of finite index in $E^{\prime}$, the set $T$ of all torsion elements of $C_{E^{\prime}} E$, is a subgroup of $E^{\prime}([\mathbf{1}])$. Since $Z(E)$ is torsion-free, $T \cap Z(E)=\{1\}$ and hence, $T$ can be considered as a subgroup of $H$. Moreover, $(\psi \circ j)(T)=$ $\{1\}$ and, since $\psi$ is injective, $j(T)=\{1\}$. The minimality of $H$ now implies that $T=\{1\}$ or $1 \rightarrow E \rightarrow E^{\prime} \rightarrow H \rightarrow 1$ is admissible.

3.2. Geometric approach. Let $\mathcal{E}(M)$ denote the space of self-homotopy equivalences of an aspherical manifold $M$. It is well-known that $\pi_{0}(\mathcal{E}(M))$ is isomorphic to $\operatorname{Out}\left(\pi_{1}(M)\right)$. We write $\Phi$ for the natural homomorphism $\Phi: \mathcal{H}(M) \rightarrow \operatorname{Out}\left(\pi_{1}(M)\right)$.

Assume from now on that $M$ is a model aspherical manifold associated to an $L$-set of data $(N, E, \rho, W)$. Write $\mathcal{H}^{f}(M)$ for the group of homeomorphisms of $M$ whose liftings to the universal cover $L \times W$ belong to $\mathcal{H}^{f}(L \times W)$. Elements of $\mathcal{H}^{f}(M)$ are called the fiber preserving homeomorphisms of $M$.

We say that an abstract kernel $\psi: G \rightarrow \operatorname{Out}(E)$ can be (effectively) geometrically realized by a group of fiber preserving homeomorphisms of $M$ if and only if there exists an (injective) homomorphism $\varphi: G \rightarrow \mathcal{H}^{f}(M)$ such that $\Phi \circ \varphi=\psi$.

It is known that a finite abstract kernel $\psi: G \rightarrow \operatorname{Out}(E)$ can be (admissibly) algebraically realized if there exists an (effective) geometric realization of $\psi([\mathbf{6}])$. While studying if the converse also holds, the following notion (terminology initially due to F. Raymond) turned out to be crucial:

Definition 3.4. An $L$-set of data $(N, E, \rho, W)$ is finitely extendable if and only if for each extension $1 \rightarrow E \rightarrow E^{\prime} \rightarrow G \rightarrow 1$ of $E$ by some finite group $G, N$ is normal in $E^{\prime}$ and $\rho: E / N \rightarrow \mathcal{H}(W)$ lifts to an action $\rho^{\prime}: E^{\prime} / N \rightarrow \mathcal{H}(W)$ such that $\left(N, E^{\prime}, \rho^{\prime}, W\right)$ is an $L$-set of data.

For example, if $N$ is characteristic in $E, W$ is a point, $\rho$ is the trivial action and, for any finite group $F^{\prime}, \rho^{\prime}: F^{\prime} \rightarrow \mathcal{H}(W)$ has the $L$-Seifert property, then each $L$-set of data $(N, E, \rho,\{\cdot\})$ is automatically finitely extendable. In other words, flat Riemannian manifolds, infra-nilmanifolds and infra-solvmanifolds of type $(\mathrm{R})$ are modelled on a finitely extendable set of data. In this perspective, the following theorem nicely generalizes similar results, for these subclasses of model aspherical manifolds, which were already presented before in the literature (respectively in [6], [3] and [5]). 
Theorem 3.5. Let $M$ be a model aspherical manifold associated to a finitely extendable $L$-set of data $(N, E, \rho, W)$. Then, for each finite abstract kernel $\psi: G \rightarrow \operatorname{Out}(E), \psi$ can be (admissibly) algebraically realized if and only if $\psi$ can be (effectively) geometrically realized.

For the proof of the theorem, we refer to [9]. We also note that it is still an open question if the above property holds for all (model) aspherical manifolds.

3.3. Main results. Although a finite abstract kernel $\psi: G \rightarrow \operatorname{Out}\left(\pi_{1}(M)\right)$ may not admit an extension of $\pi_{1}(M)$ by $G$, it is possible to "enlarge" $G$ to another finite group $H$ whose resulting abstract kernel $H \rightarrow \operatorname{Out}\left(\pi_{1}(M)\right)$ admits a geometric realization:

Theorem 3.6. Let $M$ be a model aspherical manifold associated to a finitely extendable set of data $(N, E, \rho, W)$. Assume $Z(E)$ is finitely generated. Then, for each (faithful) finite abstract kernel $\psi: G \rightarrow \operatorname{Out}(E)$, there is a finite group $H$ and an epimorphism $j: H \rightarrow G$ such that the abstract kernel $\psi \circ j: H \rightarrow \operatorname{Out}(E)$ can be (effectively) geometrically realized.

Proof. One part of the proof is a straightforward consequence of Proposition 3.2 and Theorem 3.5. If moreover $\psi: G \rightarrow \operatorname{Out}(E)$ is faithful, then one can reduce to a minimal inflation $j: H \rightarrow G$ and because of Lemma 3.3 and Theorem $3.5, \psi \circ j$ can be effectively geometrically realized.

This theorem also generalizes analogous results for flat Riemannian manifolds ([6], [12]) and infra-nilmanifolds ([2]). In fact, in [6], it was conjectured that this theorem might hold for all aspherical manifolds. In this perspective, it is important to note that Theorem 3.6 provides a positive answer to this conjecture in already a quite general set-up.

The reader should also remark that the assumption in Theorem 3.6 of finite generation of the center of $\pi_{1}(M)$, is needed in the proof but, to the best of our knowledge, at this moment no examples of aspherical manifolds $M$ are known where $Z\left(\pi_{1}(M)\right)$ is not finitely generated.

Anyhow, Theorem 3.6 is the key observation to prove the following result, which seems to be the most natural way of solving the failure of Nielsen's theorem.

Theorem 3.7. Let $M$ be model aspherical manifold associated to a finitely extendable set $(N, E, \rho, W)$ and assume that $Z(E)$ is finitely generated. Then each homotopically periodic continous self-map $f$ of $M$ is homotopic to a fiber preserving homeomorphism $g$ of $M$ of finite order.

In particular, if the homotopy period of $f$ is $k$, the order of $g$ is $l$ and if the rank of $Z(E)$ is $n$, then $k \leq l$ and $l$ divides $k^{n+1}$.

Proof. Let $f: M \rightarrow M$ be homotopically periodic or, for some $k \in \mathbb{N}_{0}$, $f^{k}$ is homotopic to the identity. Assume that for each $k^{\prime}<k, f^{k^{\prime}}$ is not 
homotopic to the identity (or $k$ is the homotopy period of $f$ ). Because $f$ and $f^{k-1}$ are homotopy inverses of each other, it follows that $f$ is a selfhomotopy equivalence or $f \in \mathcal{E}(M)$. So, $f$ induces a faithful abstract kernel $\psi: \mathbb{Z}_{k} \rightarrow \pi_{0}(\mathcal{E}(M)) \cong \operatorname{Out}(E)$.

Because of Theorem 3.6, there exists a finite group $H$ and an epimorphism $j: H \rightarrow \mathbb{Z}_{k}$ such that $\psi \circ j: H \rightarrow \operatorname{Out}(E)$ can be effectively geometrically realized, say via $\varphi: H \rightarrow \mathcal{H}^{f}(M)$. Choose $h \in H$ such that $\psi(j(h))=$ $\Phi(\varphi(h))$ is the homotopy class of $f$. In other words, $g=\varphi(h) \in \mathcal{H}^{f}(M)$ and $f$ induce the same outer automorphism of $E$ or $g$ and $f$ are homotopic. And because $H$ is finite, $g$ is of finite order, say $l$. Obviously, $k \leq l$. Moreover, the order of the obstruction corresponding to $\psi: \mathbb{Z}_{k} \rightarrow \operatorname{Out}(E)$ divides $k$ and hence the order of $H$ divides $k^{n+1}$ (Proposition 3.2).

Observe that if $\pi_{1}(M)$ is centerless (or $n=0$ ), then Nielsen's theorem does hold for this manifold $M$.

For model aspherical manifolds modelled on an $L$-set of data $(N, E, \rho,\{\cdot\})$ (with $\rho$ the trivial action), the Seifert construction can be done smoothly. In this case, the group of fiber preserving diffeomorphisms of $L$ compatible with left translations is $L^{*} \rtimes \operatorname{Aut}(L)$, which is isomorphic to the classical affine group $\operatorname{Aff}(L)=L \rtimes \operatorname{Aut}(L)$ of $L$ (via $L^{*} \rtimes \operatorname{Aut}(L) \rightarrow \operatorname{Aff}(L)$ sending $\left.(l, g) \mapsto\left(l, \mu\left(l^{-1}\right) g\right)\right)$. The group of diffeomorphisms of $M$ whose liftings to the universal cover $L$ belong to $\operatorname{Aff}(L)$ is referred to as $\operatorname{Aff}(M)$, the group of affine diffeomorphisms of $M$ onto itself. Hence:

Corollary 3.8. If $M$ is a flat Riemannian manifold, an infra-nilmanifold or an infra-solvmanifold of type (R), then each homotopically periodic selfmap of $M$ is homotopic to an affine diffeomorphism of $M$ of finite order.

To be complete, we have to mention that this result, more specifically for infra-nilmanifolds, is implicitly present in [4].

\section{Example}

In the following example, we use an infra-nilmanifold $M$ with $Z\left(\pi_{1}(M)\right) \neq\{1\}$ and illustrate our weaker version of Nielsen's theorem (Theorem 3.7).

Example 4.1. Consider the Heisenberg group

$$
L=\left\{\left(\begin{array}{ccc}
1 & y & z \\
0 & 1 & x \\
0 & 0 & 1
\end{array}\right) \mid x, y, z \in \mathbb{R}\right\}
$$

which is a connected, simply connected, 2-step nilpotent Lie group. Take

$$
A=\left(\begin{array}{lll}
1 & 0 & 0 \\
0 & 1 & 1 \\
0 & 0 & 1
\end{array}\right), \quad B=\left(\begin{array}{lll}
1 & 1 & 0 \\
0 & 1 & 0 \\
0 & 0 & 1
\end{array}\right), \quad C=\left(\begin{array}{lll}
1 & 0 & 1 \\
0 & 1 & 0 \\
0 & 0 & 1
\end{array}\right)
$$


such that $L=\left\{A^{x} B^{y} C^{z} \| x, y, z \in \mathbb{R}\right\}$. Observe that $[B, A]=C,[C, A]=1$ and $[C, B]=1$.

Take the lattice $N$ in $L$ generated by $a=A, b=B$ and $c=C^{\frac{1}{k}}$ (for some $k \in \mathbb{Z}_{0}$ ). As a presentation for $N$, we have

$$
N:\left\langle a, b, c \|[b, a]=c^{k},[c, a]=[c, b]=1\right\rangle .
$$

Let $F \cong \mathbb{Z}_{2}$, given as $\{1, \alpha\}$, act on $L$ via $\varphi: F \rightarrow \operatorname{Aut}(L)$ such that $\alpha$ sends $A \mapsto A^{-1}, B \mapsto B^{-1}$ and $C \mapsto C$. Let $E$ be presented by

$$
\begin{array}{rllll}
E:\langle a, b, c, \alpha \| & {[b, a]=c^{k}} & {[c, a]=1} & {[c, b]=1} & \\
\alpha a=a^{-1} \alpha & \alpha b=b^{-1} \alpha & \alpha c=c \alpha & \left.\alpha^{2}=c\right\rangle .
\end{array}
$$

Clearly, $E$ fits into an extension $1 \rightarrow N \rightarrow E \rightarrow \mathbb{Z}_{2} \rightarrow 1$ and $Z(E) \cong \mathbb{Z}$, the subgroup generated by $c$. A Seifert construction $\Psi: E \rightarrow \operatorname{Aff}(L)=$ $L \rtimes \operatorname{Aut}(L)$ for this set of data is given by $a \mapsto(A, 1), b \mapsto(B, 1), c \mapsto\left(C^{\frac{1}{k}}, 1\right)$ and $\alpha \mapsto\left(C^{\frac{1}{2 k}}, \varphi(\alpha)\right)$. If we from now on assume that $k$ is even, then (and only then) $E$ is torsion-free and the corresponding model aspherical manifold $M=E \backslash L$ is a 3 -dimensional infra-nilmanifold.

Take $\tilde{f}=(\sqrt{A} \sqrt{B}, \nu) \in \operatorname{Aff}(L)$ where $\nu \in \operatorname{Aut}(L)$ sends $A \mapsto A, B \mapsto B^{-1}$ and $C \mapsto C^{-1}$. Obviously, $\nu$ and $\varphi(\alpha)$ commute and the conjugation $\mu(\tilde{f})$ in $\operatorname{Aff}(L)$ sends $\Psi(a) \mapsto \Psi\left(a c^{\frac{k}{2}}\right), \Psi(b) \mapsto \Psi\left(b^{-1} c^{\frac{k}{2}}\right), \Psi(c) \mapsto \Psi\left(c^{-1}\right)$ and $\Psi(\alpha) \mapsto \Psi\left(a b c^{\frac{k}{2}-1} \alpha\right)$. Hence $\tilde{f}$ defines an affine diffeomorphism $f$ of $M$ and an automorphism $f_{*}$ of $E$ sending $a \mapsto a c^{\frac{k}{2}}, b \mapsto b^{-1} c^{\frac{k}{2}}, c \mapsto c^{-1}$ and $\alpha \mapsto$ $a b c^{\frac{k}{2}-1} \alpha$. The reader verifies that the corresponding outer automorphism $\Phi(f)$ is of order 2 in $\operatorname{Out}(E)$ since $f_{*}^{2}=\mu(a)$. We conclude that $f: M \rightarrow M$ is of homotopy period 2 .

Because of Lemma 3.1, the faithful abstract kernel $\psi: \mathbb{Z}_{2} \rightarrow \operatorname{Out}(E)$ induced by $f$ can be algebraically realized (and hence effectively geometrically) if and only if $k \equiv 0 \bmod 4: f_{*}^{2}=\mu(x)$ if and only if $x=a c^{z}$, for some integer $z$, and $f_{*}(x)\left(=a c^{\frac{k}{2}-z}\right)=x$ if and only $z=\frac{k}{4}$. In case $k \not \equiv 0 \bmod 4$, then the "enlarged" abstract kernel $H \cong \mathbb{Z}_{4} \rightarrow \operatorname{Out}(E)$, sending $1 \mapsto f_{*} \operatorname{Inn}(E)$ (which is not injective), can be algebraically realized (again use Lemma 3.1): $f_{*}^{4}=\mu(x)$ and $f_{*}(x)=x$ if we take $x=a^{2} c^{\frac{k}{2}}$. Obviously, $H$ is minimal or this abstract kernel $\mathbb{Z}_{4} \rightarrow \operatorname{Out}(E)$ can be effectively geometrically realized (Theorem 3.6). We conclude that $f$ is homotopic to an affine diffeomorphism of $M$ of order 2 if $k \equiv 0 \bmod 4$ and if $k \not \equiv 0 \bmod 4$ then $f$ is homotopic to an affine diffeomorphism of order 4 (which illustrates Theorem 3.7).

And indeed, observe that $f: M \rightarrow M$ sends the orbit of an element $A^{x} B^{y} C^{z}$ of $L$ to the point of $M$ represented by

$$
\tilde{f}\left(A^{x} B^{y} C^{z}\right)=\sqrt{A} \sqrt{B} \nu\left(A^{x} B^{y} C^{z}\right)=A^{\frac{1}{2}+x} B^{\frac{1}{2}-y} C^{\frac{x}{2}-z} .
$$

Hence, $f^{2}$ sends the point of $M$ represented by $A^{x} B^{y} C^{z}$ to the orbit of $A^{x+1} B^{y} C^{z+\frac{1}{4}}$, which is also represented by $A^{x} B^{y} C^{z+\frac{1}{4}}$. Therefore, $f \in$ 
$\operatorname{Aff}(M)$ is of order 2 if and only if $k \equiv 0 \bmod 4$ and if $k \not \equiv 0 \bmod 4$, then $f$ is only of order 4 .

\section{References}

[1] K. Dekimpe, A note on the torsion elements in the centralizer of a finite index subgroup, Bull. of Belg. Math. Soc., 3 (1996), 497-500.

[2] P. Igodt, Generalizing a realization result of B. Zimmermann and K.B. Lee to infranilmanifolds, Manuscripta Math., 47 (1984), 19-30.

[3] Y. Kamishima, K.B. Lee and F. Raymond, The Seifert construction and its applications to infra-nilmanifolds, Quarterly J. of Math. Oxford, 34(2) (1983), 433-452.

[4] S. Kwasik and K.B. Lee, The Nielsen numbers of homotopically periodic maps of infra-nilmanifolds, J. London Math. Soc., 38(2) (1988), 544-554.

[5] K.B. Lee, Infra-solvmanifolds of type (R), Quart. J. Math. Oxford, 46(2) (1995), 185-195.

[6] K.B. Lee and F. Raymond, Topological, affine and isometric actions on flat Riemannian manifolds, J. Differential Geometry, 16 (1981), 255-269.

[7] _ Topological, affine and isometric actions on flat Riemannian manifolds II, Topology and its Applications, 13 (1982), 295-310.

[8] _ Seifert manifolds modelled on principal bundles, Transf. Groups (Osaka, 1987); Lect. Notes in Math., 1375 (1989), 207-215.

[9] W. Malfait, Nielsen's theorem for model aspherical manifolds, Manuscripta Math., 90 (1996), 63-83.

[10] _ Algebraic criteria to decide if a finite group acts effectively on a model aspherical manifold, Journal of Algebra, 206 (1998), 51-66.

[11] F. Raymond and L.L. Scott, Failure of Nielsen's theorem in higher dimensions, Arch. Math., 29 (1977), 643-654.

[12] B. Zimmermann, Über Gruppen von Homöomorphismen Seifertscher Faserraume und flacher Mannigfaltigkeiten, Manuscripta Math., 30 (1980), 361-373.

Received May 7, 1998. The author is Postdoctoral Fellow of the Fund for Scientific Research - Flanders (Belgium) (F.W.O.).

Katholieke Universiteit LeUVEN

B-8500 KORTRIJK

BELGIUM

E-mail address: Wim.Malfait@kulak.ac.be 